\title{
EXOTIC FRUITS AND VEGETABLE FOOD AS NUTRITIONAL SUPPLEMENT FOR DIABETES, OBESITY AND METABOLIC DISEASES
}

\author{
SHRADDHA JOSHI*, VARSHA JADHAV, VILASRAO KADAM \\ Bharati Vidyapeeth's College of Pharmacy, C. B. D. Belapur, Navi Mumbai 400614 \\ Email: shraddha25.sj@gmail.com
}

Received: 26 Dec 2017, Revised and Accepted: 05 Feb 2018

\begin{abstract}
The most challenging diseases such as obesity and diabetes are growing wider and its treatment is a major challenge for healthcare professionals. Most of these metabolic diseases are because of the impact of lifestyle on health. Many studies have recognized a high intake of fruits, greens and other vegetables, nuts and pulses play an important role in our daily diet. Biological constituents are important to combat such diseases and can act as nutritional supplements for treatment of diabetes, cancer, obesity and cardiovascular diseases. The aim of this article is to explore the role of all these exotic fruits and vegetable food in the pathophysiology of metabolic diseases and in alteration of diabetes and obesity.
\end{abstract}

Keywords: Exotic fruits, Vegetable food, Diabetes, Obesity, Nutritional supplement

(C) 2018 The Authors. Published by Innovare Academic Sciences Pvt Ltd. This is an open-access article under the CC BY license (http://creativecommons.org/licenses/by/4.0/) DOI: http://dx.doi.org/10.22159/ijcpr.2018v10i2.25843

\section{INTRODUCTION}

The ubiquity of type 2 diabetes and obesity is growing rapidly and its growth is a major provocation for healthcare professionals. Obesity is identified by gathering of excess fat in adipose tissue and thus serves as a trigger for the host for various multi-organ issues, from cardiovascular diseases to cancer. The most preventive therapy is to intake low energy diet and healthy food, as well as physical activity to combat such metabolic diseases [1].

Whereas, Diabetes mellitus (type 2), is a long-term disorder that is defined by characteristics such as insulin resistance, high blood sugar and relative lack of insulin. These leads to health complications from high blood sugar which include strokes, heart diseases, diabetic retinopathy which can lead to kidney failure, blindness and poor blood flow in the limbs which results in amputations. Noninsulin-dependent diabetes is a chronic condition that affects the way your body metabolizes glucose, causes lack of production of insulin to maintain a normal glucose level [2].

Recently, the bioactive components in fruits and functional food have been used as the therapeutic complement in the treatment of chronic diseases. In India, Pakistan as well as in ancient Chinese medicine includes locally harvested fruits which have various therapeutic activities such as antioxidant, an anti-inflammatory which will directly improve metabolic syndrome. The word 'vegetable food' collectively includes a variety of food items, such as leafy vegetables, tomato, pumpkin, aromatic plants and spices, pulses, starchy food and nuts. Besides many nutrients, it also includes some bioactive constituents which show a variety of activity. In this regard, pulses play an important role in weight control whereas, the flavonoid content has chemoprotective and anti-cancer properties [3].

Hence, we believe that extremely important that the global scientific community should further analyse the importance of this vegetable food in the pathogenesis of obesity, diabetes and other metabolic diseases [2].

\section{Bio defensive mechanism}

\section{Exotic fruits}

Fruits are an important ingredient of our daily diet which consists of multiple bioactive nutraceuticals, which enhance our body's strength to fight against various diseases. Some of them are described below.

\section{LITHI: (Litchi chinensis)}

Originally from Southeast Asia.

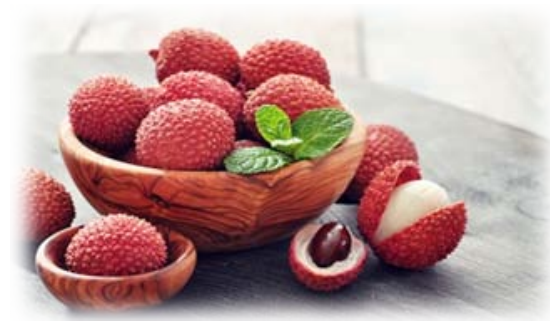

Feeding of litchi extract in rats shows an enhanced rate of metabolic profile, characterized by decreased body weight, total cholesterol, triglycerides, fasting glucose and fasting insulin level.

The anti-inflammatory effect of the extract is responsible for showing anti-diabetic activity. Also, the litchi fruit pulp and its constituents contain potent inhibitors of aldose reductase, which is a major reason to trigger glucose-induced cataract, thus fruit pulp can be used as prevention for diabetes-induced retinopathy and blindness. (4) The fruit extract also shows higher scavenging activity against DPPH radical, hydroxyl and superoxide radical. The polyphenol content in fruit has marked promise in preventing and alleviating obesity-induced metabolic syndrome [2].

\section{Durian: (Durio zibenthinus)}

Widely distributed in south-east Asia and is considered as "the king of fruits."

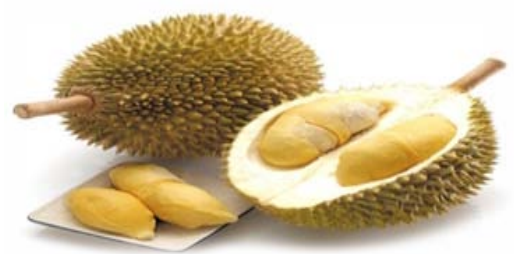

Durian exhibits potential anti-diabetic and anti-obesity activities due to the presence of various metabolic enhancer and antioxidant Interestingly, in the small clinical trial, durian has shown to improve glucose homeostasis by altering insulin secretion and its action [5] The anti-oxidant property of durian constituents can be useful for 
prevention of oxidative stress-mediated induction of diabetic and obesity complications.

Daily consumption of fresh fruit is important as it contains healthpromoting bioactive component such as anthocyanins, flavonoids, vitamins and minerals which has anti-oxidant activity. Durian flesh, as well as skin, has found to be anti-diabetic, anti-hyperlipidemic, antiproliferative and anti-microbial activity [6].

\section{Jackfruit: (Artocarpusheterophyllus)}

Native to the Western Ghats in India and is widely prevalent throughout the Southeast Asian region [7]

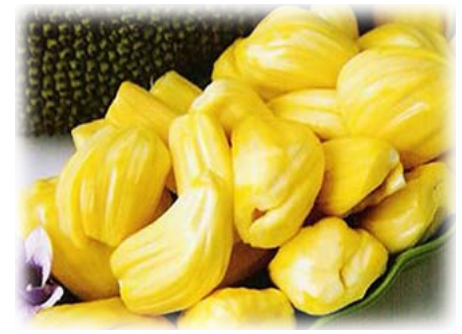

Presence of flavonoids, stilbenoids in the extract of jackfruit significantly improved glucose tolerance in both normal as well as diabetic patients. (2) The phenolic content Artocarpesin is responsible for anti-inflammatory activity by suppressing lipopolysaccharide-induced by nitric oxide (NO) and prostaglandin E2 (PGE2). Interestingly, the jackfruit seeds demonstrate higher anti-oxidant capacities than edible portion [8]

\section{Mangosteen: (Garcinia mangostana)}

Grows on tropical trees in India, Myanmar, Malaysia, Philippines, Sri Lanka and Thailand.

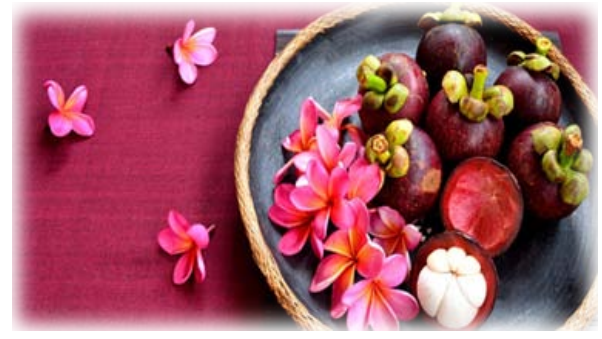

It is a medicinal plant, popularly used for diabetic complications and its induced obesity. The XanGo juice of these fruit exhibit potential anti-inflammatory activity by significantly reduced C-reactive protein levels. Mangostin, one of the most potent xanthones, exhibited a potential free radical scavenging property and protected oxidation of Low-density lipoprotein (LDL) [9].

\section{ACAI: (Euterpe oleracea)}

Grows on a large palm tree indigenous to South America.

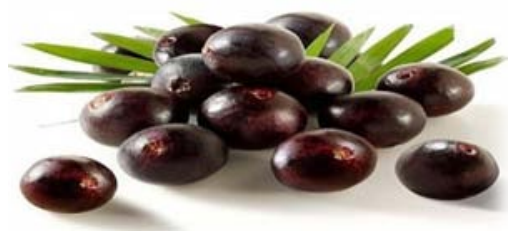

It is a dark, purple, berry-like fruit and has been widely used as a medicinal agent in ancient times. Administration of acai pulp in female Fischer rats, shows reduced total and non-high-density lipoprotein cholesterol, suggesting a clear hypercholesterolemic effect. Acai possesses anti-inflammatory and anti-oxidant properties. Supplementation of acai to hypercholesterolemic diet significantly decreased serum levels of end products of oxidative stress [2].

Acai contains a higher amount of. anthocyanin and polyphenolic content which synergistically acts as anti-oxidant capacity, whereas cyanidin-3-glucoside was the principal anthocyanin that contributes to the antioxidant capacity. Acai was found to be potential inhibitors of COX-1 and COX-2, thus possess antiinflammatory activity [2].

\section{GOJI: (Lyciumbarbarum)}

Native to East Asia.

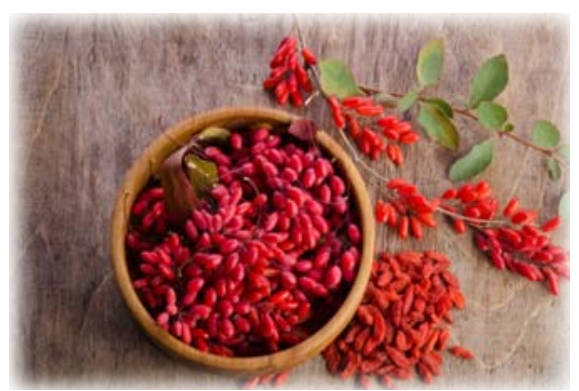

It is most commonly used in Traditional Chinese medicine to attenuate a spectrum of health ailments including diabetes and obesity. Goji fruit appears to have promising therapeutic application for diabetes, obesity and resultant complications. Goji fruit's extract of crude polysaccharide and purified polysaccharide fractions markedly reduced blood glucose levels, serum total cholesterol and triglyceride content and increased HDL levels in alloxan-induced diabetic rabbits. It has been found that goji fruit administration decreased plasma insulin levels, body weight and increased the insulin-sensitive index in diabetic rats [2].

Goji fruit also possesses potent antioxidant and cardioprotective effects. Further, goji polysaccharide treatment $(10 \mathrm{mg} / \mathrm{kg})$ results in decreased levels of malondialdehyde (MDA), NO and blood glucose. Thus, the study suggests that goji is able to regulate glucose metabolism and homeostasis and provide protection from diabetesinduced oxidative stress-mediated complications [10].

\section{Pomogranate: (Punicagranatum L.)}

An ancient fruit native to regions from the Himalayas in northern India and Iran [7].

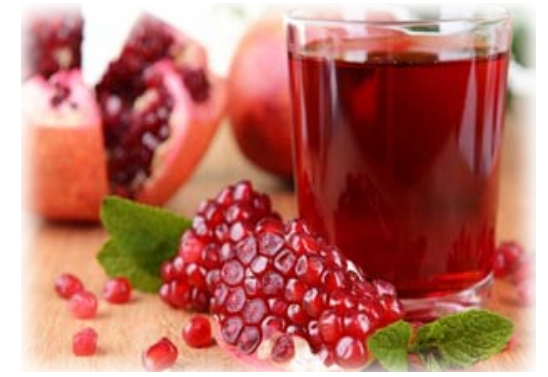

Pomegranate has been commonly used in various ancient medicine, such as Ayurveda, for treatment of diabetes. A study in male mice showed the consumption of pomegranate seed oil reduced weight gain and improved insulin sensitivity suggesting a diminished development to type-2 diabetes. Experiments in 3T3-L 1 preadipocytes suggest that punicic acid, activates peroxisome proliferator-activated receptor-gamma (PPAR- $\gamma$ ), an important target of insulin action and energy metabolism, and alleviate pathogenesis of obesity and diabetes through PPAR- $\gamma$ mediated mechanism [11].

More recently, studies show that pomegranate juice extract may prevent High Blood Pressure induced by Angiotensin II in diabetic rats by ameliorating oxidative stress and inhibiting Angiotensinconverting enzyme (ACE) activity. The basis of this study can be suggested that pomegranate can be considered as a rational complementary therapeutic agent to combat obesity, diabetes and the resultant metabolic syndrome [2].

\section{AVOCADO: (Persea americana)}

A fruit native to the Caribbean, Mexico, South America and Central America. 


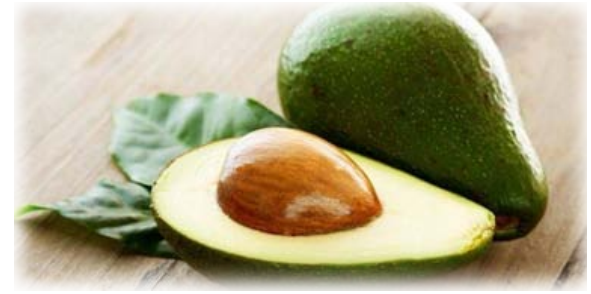

Surprising data found in a clinical trial that, feeding of 55 subjects with $200 \mathrm{~g} /$ day of avocado for $6 \mathrm{w}$ with an energy-restricted diet dramatically decreased body weight, body mass index and percentage body fat. Uncommonly, the constituents present in avocado such as MUFA's are responsible agents showing an improved lipid profile and adequately maintained glycemic control in type 2 diabetic patients.

Furthermore, anti-inflammatory and antioxidant capacities of avocado are well established, indicating that inclusion of avocado into diet might ameliorate the pathogenesis of metabolic syndrome. However, it is important to note that an optimal quantity of avocado must be consumed for the observed anti-obese and anti-diabetic effects [2].

\section{Green and other vegetable food}

Vegetables also supply a large number of phytochemicals-from aldehydes to alkaloids. These components have beneficial health effects, namely, chemoprotective properties. Polyphenols play an important role in showing a wide range of properties. Other characteristics include reactive oxygen species (ROS)-scavenging capacity and the ability to interact with dietary proteins [12].

\section{Cabbage: (Brassica oleraceae)}

Native to coastal southern and Western Europe.

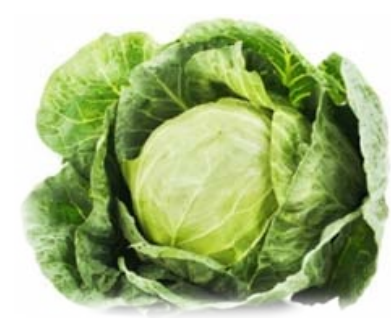

A diet rich in cruciferous vegetables (e. g. cabbage, broccoli, cauliflower) plays an important to reduced risk of several human cancers, might be associated with the presence of ROS scavengers as carotenoids, xanthophylls and flavonoids. Phytochemicals such as quercetin have significant antioxidant potential in vitro than vitamins $\mathrm{A}, \mathrm{C}$ and $\mathrm{E}$, which synergistically results in antiinflammatory and anti-cancer activities [1].

\section{Turnip: (Brassica rapa)}

Usually grown in temperate climates worldwide.

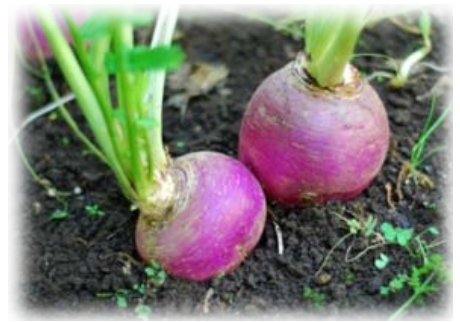

Turnip greens contain more vitamins than the root, which includes $\beta$-carotene, lutein and zeaxanthin, $\alpha$-tocopherol and higher levels of phylloquinone than the corresponding root. Turnip green also contains important flavonols: kaempferol and quercetin.

It has also been found that, as increasing energy expenditure, possibly influencing metabolic control in humans, with an impact on obesity and diabetes [12].

\section{Guar gum: (Cymopsis tetragonolobus Linn, Leguminosae)}

Native to India, Pakistan and USA.

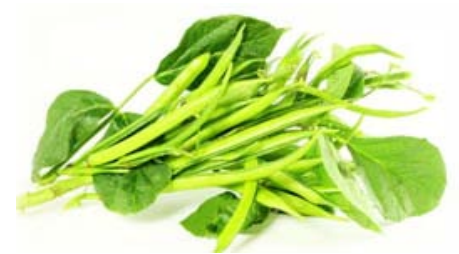

Guar gum contains water-soluble part having high molecular weight: GALACTOMANNAN commonly known as GUARAN [7].

Data obtained from the recent study revealed that administration of Guar gum for 14 consecutive days improved hyperglycemia in Streptozocin (STZ)-induced diabetic rats by a significant reduction in serum glucose level and rise in serum insulin level.Guar gum reduced insulin resistance and improved insulin sensitivity as well as $\beta$-cell function. The oral administration of petroleum ether extract significantly lowered blood glucose. The antihyperglycemic action of guar gum could be related to the ability of its fibre to produce high viscosity at low concentration in the gut lumen. This reduces the intestinal absorption of monosaccharides and disaccharides by delaying gastric emptying [13].

\section{Aromatic plants}

Aromatic plants are rich in vitamins but most particularly in flavonoids. Vitamin content plays a secondary role in the diet, due to low intake of these foods.

\section{Rosemary: (Rosmarinus officinalis)}

Rosemary is reasonably hardy in cool climates.

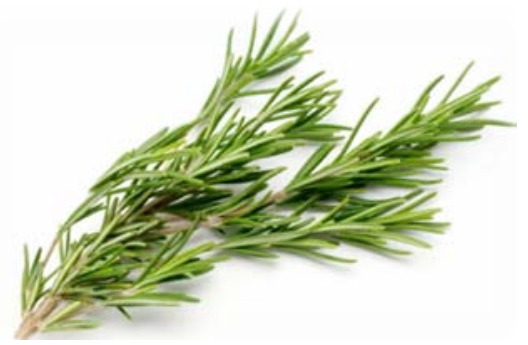

Rosemary contains many other bioactive compounds such as apigenin and luteolin, as well as naringin, readily metabolized to naringenin. Naringenin exerts a number of pharmacological effects, such as antioxidant activity, lowering blood lipid's level, anti-carcinogenic activity and in the treatment of metabolic syndrome [9].

\section{Garlic: (Allium sativum)}

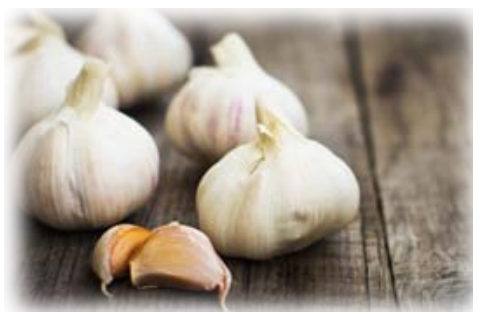

Allicin is a sulphur-containing constituent with various biological properties. Garlic contains flavonols such as kaempferol, myricetin and quercetin, which synergistically shows ROS-scavenging activities with allicin, which results in anti-ageing and chemoprotective effects. Allicin also has other pharmacological actions such as anti-infective agent, ROS scavenger, as well as act acting as hypolipidemic and hypoglycemic agent [12].

PULSES: Pulses have a distinct nutritional profile including several dietary composition factors which plays an important role in weight control. They are a good source of digestible protein and fibres with relatively low in energy, moderate to poor in fats, which includes 
mainly MUFA and PUFA. Pulse carbohydrates are slowly digested, which ascribed them some of the lowest glycemic index scores of carbohydrate-containing foods. Proteins can be more satisfactory than carbohydrates or lipids, and pulses have been implicated in providing satiety while keeping weight under control. Pulses are the good source of multiple vitamins and provide valuable constituents [12]

\section{LENTILS: (Lens culinaris)}

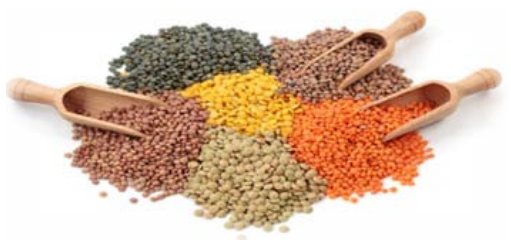

Lentils are available in a variety of size and colour, ranging from yellow and red-orange to green, brown and black. Lentils mainly contain relevant amounts of water-soluble vitamins such as thiamine, folates and other B vitamins, phylloquinone and $\gamma$ tocopherol. Lentils also contain flavonoids which have been proved to have cardioprotective effects directly or indirectly. These flavonoids and tannins such as catechin, gallocatechin having relevant health benefits such as ROS scavenging thus act as antiinflammatory and anti-carcinogenic effects. Flavonoid intake has an inverse effect on type II diabetes [12].

NUTS: Nuts have been consumed raw or used to stuff dry figs or dates, as appetizers. 'Scientific evidence proposed but does not prove that eating 1.5g ounces per day of most nuts as a part of diet low in saturated fat and cholesterol may lower the risk of cardiovascular disease.' Nuts are the fruits which comprising a hard shell and a seed, found in the variety of genera. They are a good source of vitamins and proteins which are important in maintenance, prevention and treatment of a wide range of health complications, including cardiovascular diseases and inflammatory disorders, mental and visual health, as well as play an important role in foetal and infant development [14].

\section{Walnut: (Juglans regia)}

Originally from Turkey.

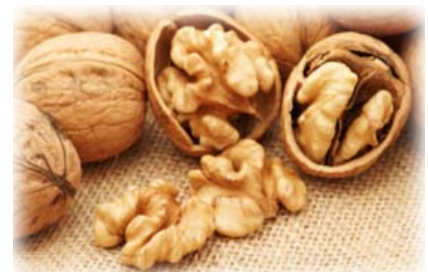

Walnut contains proteins, fats, MUFA and PUFA, as well as dietary fibre. Walnuts contain the B vitamins: thiamine, niacin, ribdlavin, pantothenic acid, folates and vitamin B6, as well as phylloquinone. Phytosterols play an important role in lowering of LDL cholesterol levels in the bloodstream. A recent study reported that dietary supplement with walnuts shows significant reduction in total cholesterol and LDL cholesterol concentration, with no untoward effects on body weight, showing effects on type-2 diabetes [9].

The fatty acid profile mainly includes MUFA and PUFA, combined with antioxidant properties of $\gamma$-tocopherol, explain the beneficial effects of nuts in relation to coronary heart diseases [16].

\section{ALMONDS: (Prunus dulcis)}

Native to the Arabian peninsula and western Asia [7].

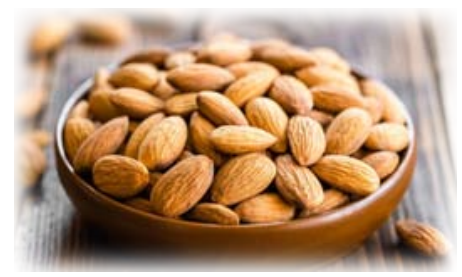

Raw almonds (with skin) supply proteins, fats, a small amount of glucose and fructose and dietary fibre. Despite this, almonds provide the $\mathrm{B}$ vitamins thiamine, rifbevin, niacin, pantothenic acid or vitamin B5, folates and vitamin B6. Many flavonoids such as catechin, epigallocatechin and epicatechin, as well as eriodictyol and naringenin. The compound eridictyol was found to increase insulinstimulated glucose uptake, shows anti-diabetic properties. Almonds are nutritionally very rich, provides a number of beneficial compounds, which shows effects such as anti-inflammatory, anticancer and antioxidant properties [16].

\section{Pine nuts: (Pinus pinea)}

Pine trees are distributed worldwide, mostly in Europe and Asia.

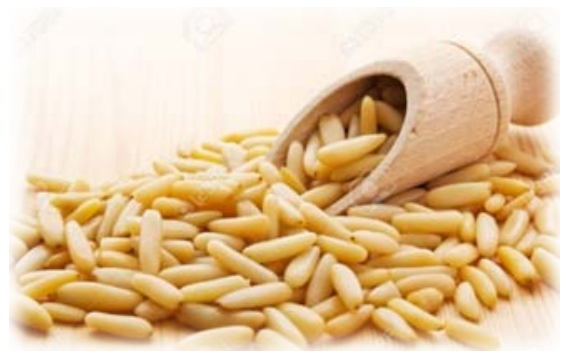

Pine nut mainly contains, proteins, fats (MUFA and PUFA) and dietary fibre. Pine nuts play an important role in controlling type II diabetes as it contains several soluble cyclitols pinitol, which may interfere with insulin sensitivity. Pine nuts suggested lowering the levels of LDL cholesterol in the blood due to the presence of wide variety of phytosterols which contributes to wellness [12].

D-pinitol influences whole-body glucose tolerance and insulin sensitivity indexes and the translocation of glucose in skeletal muscles thus suggested to have a good impact on controlling type II diabetes. Consumption of pine nut's oil also prevents oxidative stress and atherosclerosis [16].

\section{Effects on fatty liver by consumption of exotic fruits}

Fatty liver diseases are defined by the extra accumulation of fat droplets in the liver, this condition is independent of alcohol consumption and hence called as Non-Alcoholic Fatty Liver Disease (NAFLD). This condition usually occurs in conditions such as obesity and type 2-diabetes which are a major cause of hepatic insulin resistance. Hepatic insulin resistance in the major factor which is responsible to induce hyperglycemia in type 2 diabetic patients through rising in hepatic endogenous glucose production. The major cause of fatty liver disease in intake of high fat/calorie diet [17].

Therefore, it is recommended to take low calorie and high fibre diet for the healthy liver. These exotic fruits have a higher amount of fibres and other bio-constituents that can enhance liver function and thus protect the liver from obesity/diabetes-induced metabolic derangements. Recently, it was found that the combination of pomegranate seed oil (PSO) and brown marine algae (Xanthigen) significantly reduced the incidence of fatty liver disease in human subjects. The findings were also characterized by significantly decreased in body weight, hepatic fat content, triglyceride and improved liver function tests [18].

In addition to above fruits originated from all over the world, many others exotic fruits are originated from India which plays a key role in diabetes and obesity.

1. The potent therapeutic effects of Longan fruit has potent antioxidant and anti-glycated activity, hinting a role in the prevention of diabetic complications.

2. A recent study shows a potential antioxidant capacity for raw Spondius pinnata K., an exotic fruit of India.

3. A review of Kokum fruithas its evident anti-obese and antidiabetic effects. Kokum has shown to possess free radical scavenging properties and anti-lipid peroxidation as well as it will lower the circulating glucose levels in streptozotocin-induced type 2 diabetic rats [19]. 
4. the Bakul fruit, native to India, has been shown to have many antidiabetic effects; oral administration of the extract shown to have a significant decrease in blood glucose and an increase in serum insulin levels, along with other positive effects [13].

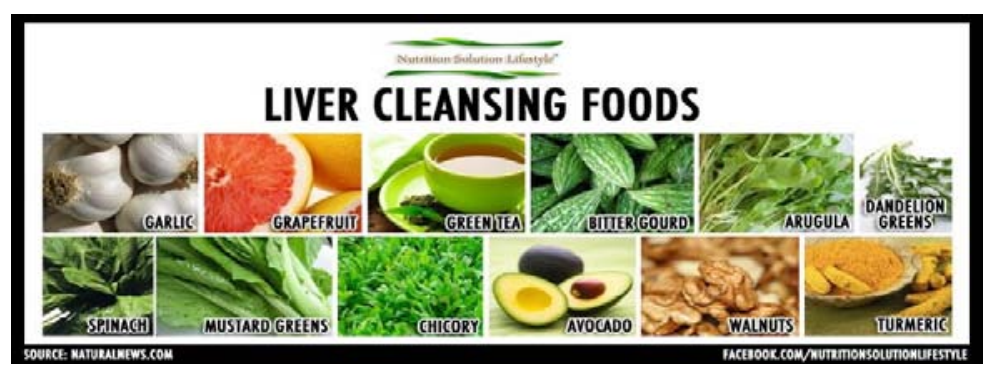

From all the information described above, we can conclude that the dietary treatment of metabolic syndrome has achieved beneficial effects on specific metabolic features such as increased plasma lipids, blood pressure and plasma glucose. Exotic fruits have various bioactive components with potential health benefits, including antiobese, anti-diabetic, anti-oxidant and anti-inflammatory. Alone or together, a combination of these health beneficial effects rendered by the reported exotic fruits is very important to prevent the development of complex pathophysiology of obesity and diabetes. The whole fruit (s) and their bio-constituents can act on various pathophysiological targets of obesity and diabetes. However, the anti-obese and anti-diabetic effects of the exotic fruits reviewed here and their bioactive constituents are clearly under-studied.

A range of different-coloured vegetables should be present on the plate: some are better raw. Aromatic herbs and spices provide flavour, anti-microbial protection for foods and important phytochemicals. Pulses are a sustainable and healthy alternative to the animal protein source, contributing to the reduction of meat consumption. Walnuts have been granted health claims regarding their contribution to the control of coronary heart disease.

\section{AUTHORS CONTRIBUTIONS}

All the author have contributed equally

\section{CONFLICT OF INTERESTS}

Declared none

\section{REFERENCES}

1. Abete I. Obesity and metabolic syndrome: potential benefit from specific nutritional components. Nutr Metab Cardiovasc Dis 2011;21:B1-B15.

2. Devalaraja S, Jain S, Yadav H. Exotic fruits as therapeutic complements for diabetes, obesity and metabolic syndrome. Food Res Int 2011;44:1856-65.

3. Ceylan-Isik AF, Fliethman RM, Wold LE, Ren J. Herbal and traditional Chinese medicine for the treatment of cardiovascular complications in diabetes mellitus. Curr Diabetic Rev 2008;4:320-8.

4. Huan HB, Xu JK. The developmental pattern of fruit tissues and their correlative relationship in Litchi chinensis Sonn. Scientia Horticultureae 1983;19:335-42.

5. Roongpisuthipong C, Banphotkasem S, Komindr S, Tanphaichitr V. Postprandial glucose and insulin responses to various tropical fruits of equivalent carbohydrate content in non- insulin-dependent diabetes mellitus. Diabetes Res Clin Practices 1991;14:123-31.

6. Lee-Hoon Ho, Bhat R. Exploring the potential nutraceutical values of durian (Duriazibethinus L.)-an exotic tropical food. Food Res Int 2014;44:81-5.

7. Shah, Biren N. Textbook of Pharmacognosy and phytochemistry. Elsevier India; 2009.

8. Jagtap UB, Bapat VA. Artocarpus: a review of its traditional uses, phytochemistry and pharmacology. J Ethnopharmacol 2010;129:142-66.

9. Banel DK, Hu FB. Effects of walnut consumption on blood lipids and other cardiovascular risk factors: a meta-analysis and systematic review. Pubmed/Am J Clin Nutr 2009;90:56-63.

10. Potterat 0 . Goji (Lyciumbarbarum and L. chinense): Phytochemistry, pharmacology and safety in the perspective of traditional uses and recent popularity. Planta Med 2010;76:7-19.

11. Hontecillas R, O'Shea M, Einerhand A, Diguardo M, BassaganyaRiera J. Activation of PPAR gamma and alpha by punicic acid ameliorates glucose tolerance and suppresses obesity-related inflammation. J Am College Nutr 2009;28:184-95.

12. Delgado, Martins A, Parisi S, Daniel M, Vaz Almeida. Greens and other vegetable foods" chemistry of the mediterranean diet. Springer Int Publishing; 2017. p. 59-137.

13. Nasry MR, AM Abo-Youssef, HA Abd El-Latif. Anti-diabetic activity of the petroleum ether extract of Guar gum in streptozotocin-induced diabetic rats: a comparative study. Beni-Suef University J Basic Appl Sci 2013;2:51-9.

14. Udani JK, Singh BB, Barrett ML, Singh VJ. Evaluation of mangosteen juice blend on biomarkers of inflammation in obese subjects: a pilot, dose-finding study. Nutr J 2009;8:48.

15. Lee YA, Cho EJ, Yokozawa T. Effects of proanthocyanidin preparations on hyperlipidemia and other biomarkers in a mouse model of type 2 diabetes. J Agric Food Chem 2008;56:7781-9.

16. Chang, Sui Kiat. Nuts and their co-products: the impact of processing (roasting) on phenolics, bioavailability, and health benefits-a comprehensive review. J Functional Foods 2016;26:88-122.

17. Krawczyk M, Bonfrate L, Lahteenmaki L. Nonalcoholic fatty liver disease. Best Pract Res Clin Gastroenterol 2010;24:695-708.

18. Lockman KA. Nyirenda MJ. Interrelationships between hepatic fat and insulin resistance in non-alcoholic fatty liver disease. Curr Diabetes Rev 2010;6:341-7.

19. Baliha M, Bhat H, Pai R, Boloor R, Palatty P. The chemistry and medical uses of underutilized Indian fruit tree Garcinia indicaChoisy (kokum): a review. Food Res Int 2011;44:1790-9. 